1993;362:213-214.

65. Gajdusek DC. Transmissible and non-transmissible amyloidoses: autocatalytic posttranslational conversion of host precursor proteins to $\beta$-pleated configurations. J Neuroimmunol 1988;20:95-110.

66. Aranda-Anzaldo A. Possible cell-free prion replication. Med Hypotheses 1992;38:249-251.

67. Pablos-Mendez A., Netto EM, Defendini R. Infectious prions or cytotoxic metabolites. Lancet 1993;341:159-161.

68. Note for guidance. Guidelines for Minimizing the Risk of Transmitting Agents Causing Spongiform Encephalopathy via
Medicinal Products. Ad Hoc Working Party on Biotechnology/Pharmacy and Working Party on Safety Measures 01/05/1992, 111/3298/91-EN.

69. Report of a WHO consultation on public health issues related to animal and human spongiform encephalopathies. November 12-14, 1991; WHO/CDS/VHP/92.104.

70. Will RG, Ironside JW, Zeidler M, et al. A new variant of Creutzfeldt-Jakob disease in the UK. Lancet 1996;347:921-925.

71. Brown P, Preece MA, Will RG. Friendly fire in medicine-hormones, gomografts, and Creutzfeldt-Jakob disease. Lancet 1992;340:24-27.

\title{
Intrinsically Contaminated Intravenous Product
}

Gina Pugliese, RN, MS; Martin S. Favero, PhD Medical News Editors

Investigators in Madrid, Spain, have reported a common-source outbreak of Burkholderia pickettii among 46 patients in three hospitals. Among the 46 culture-positive cases, there were 48 infections ( 44 bacteremias and 2 intravenous catheter-associated infections without bacteremia). $B$ pickettii was isolated from six parenteral nutrition samples that contained ranitidine (BBL; Alonga, Madrid, Spain). Vials of ranitidine were collected for culture, and $B$ pickettii was isolated from vials in a specific lot at one hospi- tal. Thirty-three isolates were analyzed and shown to be of the same biotype, one that is associated with low virulence, which may explain why there were no deaths.

$B$ pickettii has been associated with outbreaks of nosocomial infection (bacteremia or colonization of the respiratory tract) due to contamination of intravenously administered products, "sterile" distilled water, chlorhexidine in water, respiratory therapy solutions, and intravenous catheters. Although this outbreak involved a greater number of cases than did any previously described outbreak, the findings were consistent with other reported outbreaks.
The authors hypothesized that the product may have been contaminated during the filter $(0.45$ and 0.22 micropore) sterilization process during production. The organism is known to be able to pass through membrane filters and has the capability of growing in water-based solutions. No new cases occurred once the product stopped being dispensed.

FROM: Fernandez C, Wilhelmi I, Andradas E, et al. Nosocomial outbreak of Burkholderia pickettii infection due to a manufactured intravenous product used in three hospitals. Clin Infect Dis 1996;22:1092-1095. 